\title{
Momentum Is Not Conserved
}

\author{
GuagSan Yu \\ Harbin Macro Dynamics Institute, 150066, China.
}

How to cite this paper: GuagSan Yu. (2020) Momentum Is Not Conserved. Journal of Applied Mathematics and Computation, 4(4), 261-266.

DOI: $10.26855 /$ jamc.2020.12.018

Received: November 17, 2020

Accepted: December 15, 2020

Published: December 31, 2020

${ }^{*}$ Corresponding author: GuagSan Yu, Harbin Macro Dynamics Institute, 150066, China.

Email: 1951669731@qq.com

\begin{abstract}
Because Newton's third law and Newton's second law proved to be wrong, then according to the new second law of motion and the new third law of motion, the new mechanics principle is produced, the most important and remarkable is, that the momentum is not conserved and that the energy is not conserved to be discovered. The non-conservation of momentum was first discovered accidentally in mechanical experiments. This is shocking. Because this shows that momentum is never conserved in the interaction of objects with different mass. A serious problem arises at this time, because the law of conservation of momentum is established by deriving Newton's law of motion. Then if the conservation of momentum is proved wrong, Newton's laws of motion will also be questioned. In fact, Newton's third and second laws have indeed been proven wrong. The newly proposed new third and new second laws of motion provide a perfect explanation for the non-conservation of momentum. The non-conservation of momentum has been theoretically proved.
\end{abstract}

\section{Keywords}

Energy, Force, Newton's law, New law of motion, Momentum is not conserved

\section{Introduction}

Newton's third law was proved to be wrong [1]. The new third law of motion states that the force and reaction force of an object interact with an object, in most cases, is different. The acting force and the reaction force are different, so it can be proved that the momentum is not conserved.

\section{The Discovery that Momentum is not Conserved}

No one does not know the law of conservation of momentum, which is derived from Newton's law of motion. But an amazing fact is that it is wrong!

Many videos of public physics courses have explanations and experimental demonstrations of the law of conservation of momentum. The two slide blocks collide on a slippery plane. According to the explanation, the total momentum of the two slide block does not change before and after the collision. However, unless the mass of the two slide block is the same, this explanation is fundamentally wrong.

No one has ever discovered that after two objects with different masses interact, their total momentum will change? This seems like a big joke. Because experiments show that the total momentum of the two bodies will change at this time.

As shown in Figure 1, Figure 2, and Figure 3 [2].

That is, the proof of the above phenomenon. The experiment was performed by two freely rotating rotating arms of exactly the same size, which were pushed to both sides by a released spring. Which the stress points of the rotating arms $\boldsymbol{O}_{1}$ is the full arm length, and the stress points of the rotating arms $\boldsymbol{O}_{2}$ is $\mathbf{1 / 2}$ the arm length. It can be seen from Figure 2 and Figure 3 that the rotation speed of the rotating arms $\boldsymbol{O}_{1}$ is twice that of the rotating arms $\boldsymbol{O}_{2}$. 
Because the specifications of the two rotating arms are exactly the same, it can be considered that the same two rotating arms have the same mass, and they have different rotational speeds after they interact, so the rotational linear speeds of their same parts are also different. In fact, the linear velocity of the rotating arms $\boldsymbol{O}_{1}$ is twice that of the rotating arms $\boldsymbol{O}_{2}$. The mass is the same but the linear velocity is 2 times different, so its momentum is also 2 times different, that is, its momentum sum is not zero, and the total momentum changes.

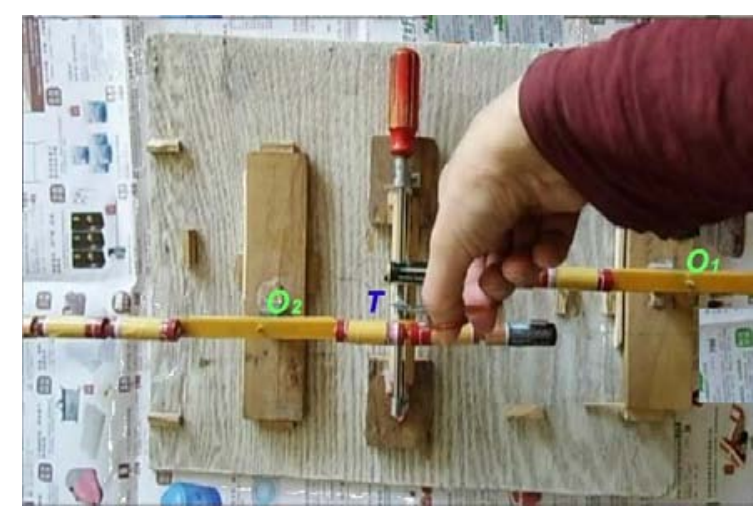

Figure 1. Rotating arm experiment.

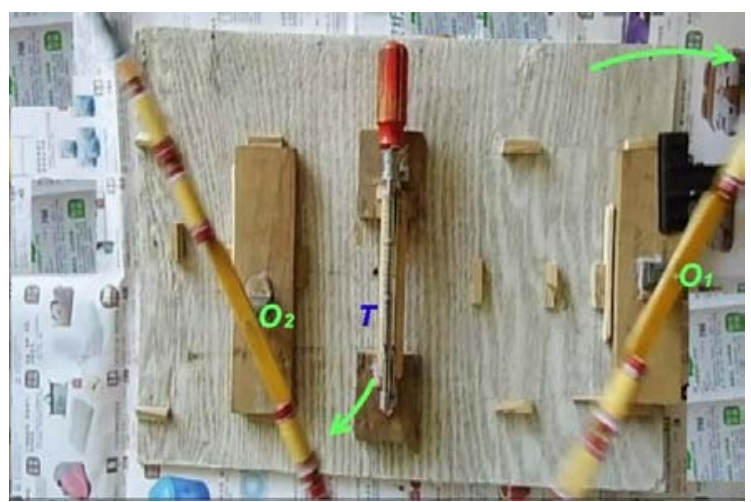

Figure 2. Angle 1 of $\mathrm{O}_{1}$ and $\mathrm{O}_{2}$.

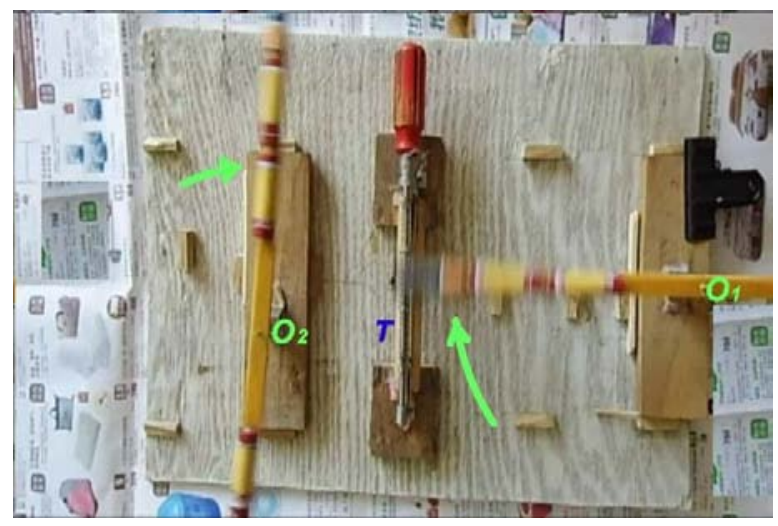

Figure 3. Angle 2 of $\mathrm{O}_{1}$ and $\mathrm{O}_{2}$.

This experiment can also be understood as that the moment arm of the rotating arm $\boldsymbol{O}_{1}$ is 2 times the moment arm of the rotating arm $\boldsymbol{O}_{2}$, therefore, the load mass of the moment arm point of the rotating arm $\boldsymbol{O}_{1}$ is $1 / 2$ of the load mass of the moment arm point of the rotating arm $\boldsymbol{O}_{2}$. And the linear velocity of the moment arm point of the rotating arm $\boldsymbol{O}_{1}$ should be 4 times the linear velocity of the moment arm point of the rotating arm $\mathbf{O}_{2}$. So the linear momentum of the moment arm points of the two rotating arms is:

That the sum of momentum is:

$$
I_{o_{1}}=\frac{m}{2} \cdot 4 u \Leftrightarrow I_{o_{2}}=m \cdot u
$$

$$
I_{o_{1}}-I_{o_{2}}=\frac{m}{2} \cdot 4 u-m \cdot u=(2-1) m \cdot u
$$

Therefore, the sum of its momentum is not zero, indicating that its total momentum has changed. The total momentum before the action is zero, and the total momentum is not zero after the action.

It is conceivable how significant the shocking meaning this experiment is.

Because the law of conservation of momentum is derived from Newton's law of motion, the law of conservation of momentum is wrong, does it mean that Newton's law of motion is also questionable?

The next series of discoveries made us even more shocked! 


\section{Find Errors in Newton's laws of motion}

The momentum of the interaction between objects of different masses is not conserved, which first reminds people of Newton's third law of motion, which may be wrong.

Trying to prove that Newton's third law of motion is wrong, and achieved results [3]. That the proof mechanism is that objects with different masses interact, and the difference in mass can be understood as the difference in space constraining force [3]. Large-mass objects have a large space constraining force and are not easy to change their motion; objects with small masses have a small spatial constraining force and are easy to change their motion.

Therefore, the acting forces generated by the two objects are different [3]. For example:

$$
\begin{array}{lc} 
& F_{a}=F_{a l l} \cdot\left(\frac{m_{a}}{m_{a}+m_{b}}\right) \\
\text { and } & F_{b}=F_{a l l} \cdot\left(\frac{m_{b}}{m_{a}+m_{b}}\right) \\
\text { and } & F_{a}=m_{b} \cdot \frac{d^{2} l_{b}}{d t^{2}} \\
\text { and } & F_{b}=m_{a} \cdot \frac{d^{2} l_{a}}{d t^{2}}
\end{array}
$$

That is, a large mass object produces a larger force to act on a small mass object, and a small mass object has a smaller force to act on a large mass object [3]. The results calculated according to the above method are completely consistent with the conditions of the aforementioned experiments.

But Newton's third law of motion was proved to be wrong, that the Newton's second law of motion it is correct? The later proof was even more shocking, and Newton's second law of motion was also proved to be wrong.

The wrong of Newton's second law of motion was discovered [1], that is based on the calculation of derivatives, on its nominal unit should be time $\boldsymbol{t}$. And as another element of survey (i.e. displacement) $\boldsymbol{l}$, it is variable. The variability of $\boldsymbol{I}$ makes the size of force $\boldsymbol{F}$ unpredictable. For example, when a spring is used to push an object, no matter how the mass of the object changes, the magnitude of the force and the distance of the force are constant. But when using Newton's second law of motion and derivative calculations, the same force acts on objects of different masses, that the acts distances are different. Therefore, if Newton's second law of motion is used to calculate the force of the spring, it will be wrong.

At this time, in order to overcome the above shortcomings, that the concept of Inverse Derivative was proposed [1] [4]. It is the opposite of the case of the derivative, which is the limit of finding the fraction when the value of the numerator approaches zero.

$$
F=\lim _{\Delta l \rightarrow 0} m \cdot \frac{\Delta l}{\Delta^{2} t^{2}}=m \cdot \frac{q l}{q^{2} t^{2}}
$$

The mathematical expression of the new second law of motion based on this principle is:

$$
F=\lim _{\Delta l \rightarrow 0} m \cdot \frac{\Delta l}{\Delta^{2} t^{2}}=m \cdot \frac{q l}{q^{2} t^{2}}=m a^{+}
$$

It is also the product of the mass and acceleration of an object. But the acceleration is calculated using the inverse derivative (denoted as $\boldsymbol{a}^{+}$) [1] [4].

Because it is an inverse derivative, although the force $\boldsymbol{F}$ and the mass $\boldsymbol{m}$ are direct proportion, the force $\boldsymbol{F}$ and the acceleration $\boldsymbol{a}^{+}$are direct ratio to the square. The relationship between the two is not simply the same ratio. Therefore, in the equivalent conversion, the acceleration $\boldsymbol{a}^{+}$must be changed by $\boldsymbol{\beta}^{2}$ times to be equivalent to the change of force $\boldsymbol{F}$ or mass $m$ by $\boldsymbol{\beta}$ times [1] [4].

So the identity transformation formula of the new second law of motion is:

$$
F=\beta m \cdot \beta^{-2} a^{+}
$$

This is very different from Newton's second law of motion, but it is only to calculate the motion of matter in this way, that the is correct. 
From the expression of the new second law of motion, it is easy to get the expression of the new third law of motion:

$$
F_{1}=m a^{+} \Leftrightarrow F_{2}=\beta m \cdot \beta^{-2} a^{+}
$$

This is the same as the previous calculation of the action and reaction force derived from the space constraining force, and the same correct result can be obtained. However, the calculations based on the expressions of the new second and new third laws of motion are completely consistent with the actual state of motion of matter. Therefore, the new second law of motion and the new third law of motion are the most correct and accurate expressions of the movement of matter.

\section{Momentum is not conserved under the new third law of motion}

From the new third law of motion, it can be directly derived that momentum is not conserved. For example:

$$
\begin{array}{ll}
\text { by } & m_{0} \cdot \frac{q l}{q^{2} t^{2}}=m_{0} a_{0}^{+}=\left.\left.F_{1}\right|_{\substack{m=m_{0} \\
a^{+}=a_{0}^{+}}} \Leftrightarrow F_{2}\right|_{\substack{m=m_{0} / \beta \\
a^{+}=\beta^{2} a_{0}^{+}}}=\frac{m_{0}}{\beta} \cdot \frac{\beta^{2} q l}{q^{2} t^{2}}=\frac{m_{0}}{\beta} \cdot \beta^{2} a_{0}^{+} \\
\text {get } & F_{1} t=m_{0} \cdot \frac{q l}{q^{2} t^{2}} \cdot t \Leftrightarrow F_{2} t=\frac{m_{0}}{\beta} \cdot \frac{\beta^{2} q l}{q^{2} t^{2}} \cdot t \\
\therefore \quad & p_{1}=m_{0} \cdot u \Leftrightarrow p_{2}=\frac{m_{0}}{\beta} \cdot \beta^{2} u=m_{0} \cdot \beta u
\end{array}
$$

Therefore, two objects interact, Objects with $\boldsymbol{\beta}$ times smaller mass, its momentum is $\boldsymbol{\beta}$ times larger. Therefore, unless the masses of the two objects are exactly the same, the two objects with different masses interact, the momentum generated by the two objects is different. Therefore, the sum of the momentum of the two bodies is not zero at this time, namely:

$$
p_{1}-p_{2}=(1-\beta)\left(m_{0} \cdot u\right) \neq 0
$$

So at this time, the total momentum of the two bodies has changed, so their momentum is not conserved.

Therefore, momentum is conserved only when the masses of the two interacting objects are exactly the same. And in nature, in most cases, the masses of interacting objects are different, so their momentum is not conserved.

So in nature, the phenomenon of non-conservation of momentum should be universal. Therefore, in classical physics, the influential law of conservation of momentum is actually wrong. It should be abolished.

Therefore, that the momentum is not conserved. Momentum is not conserved!

\section{Angular Momentum is also have Conserved and Non-Conserved}

The law of conservation of angular momentum is also an important law in classical physics. Because of the establishment of the new law of motion, the law of conservation of angular momentum will only be applicable in some special cases, and no longer applicable in other cases.

Conservation of angular momentum is applicable in the movement of a single celestial body (or the movement of a single particle around the center of the circle) [5], for example:

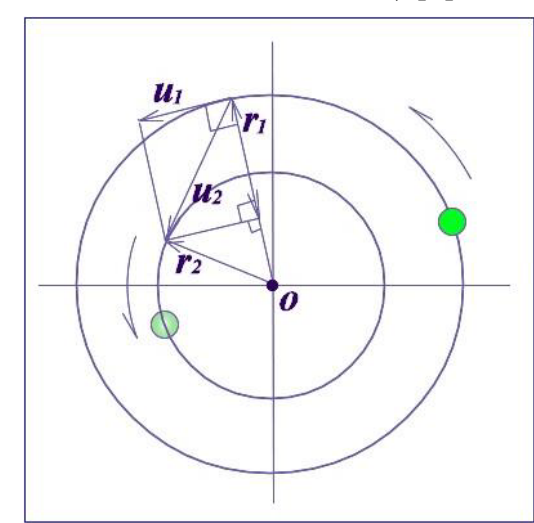

Figure 4. $\mathrm{R}$ changes from large to small.

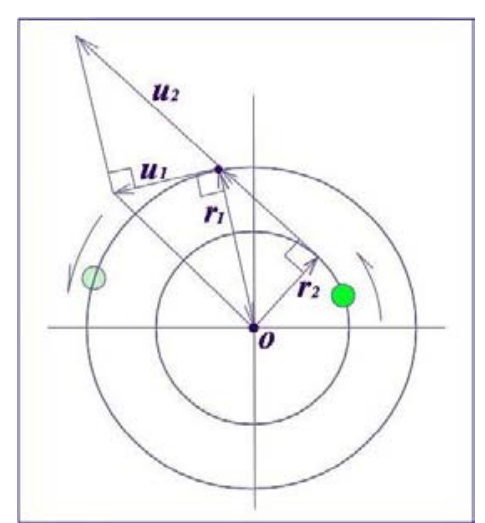

Figure 5. R changes from small to large. 
From Figure 4 and Figure 5, it can be seen that: When the radius of the circular motion of an object or particle changes, its linear velocity will change in inverse proportion. This situation is determined by the principle of vector decomposition (Figure 5) and synthesis (Figure 4). Therefore, it is a natural choice regarding the movement of matter and laws of force, which is inevitable. At this time:

$$
\frac{r_{1}}{r_{2}}=\frac{u_{2}}{u_{1}}
$$

At this time, the velocity vector and the radius of rotation change in inverse proportion, so the angular momentum of the object does not change at this time.

$$
l=m \cdot r_{1} \cdot u_{1}=m \cdot r_{2} \cdot u_{2}
$$

Therefore, the conservation of angular momentum is at this time, and the law of conservation of angular momentum applies.

In classical mechanics, the law of conservation of angular momentum is considered to be suitable for all rotating objects, including rotating particle systems. However, according to the new laws of motion [1] [3] and the new rotational dynamics [3] [5], in any complex rotating system and circumvolve system, the non-conservation of angular momentum should be universal.

According to the new rotational dynamics [5]:

$$
\begin{aligned}
& m=\frac{\left(m_{1} r_{1}+m_{2} r_{2}+\cdots m_{n} r_{n}\right)^{2}}{\left(m_{1} \cdot r_{1}^{2}+m_{2} \cdot r_{2}^{2}+\cdots m_{n} \cdot r_{n}^{2}\right)} \\
& m=\frac{I_{C}^{2}}{L_{\text {all }} / \omega_{1}}
\end{aligned}
$$

In a rotating rigid body or particle system, set $\boldsymbol{A}^{\prime}$ is move the object and set $\boldsymbol{C}$ is rotate the rigid body, the formulas (4.0.3) and (4.0.4) must be satisfied just now to satisfy the conservation of angular momentum. But in any complex rigid body system and particle system, it is almost impossible to fully satisfy the above two formulas. Therefore, in most cases, for a complex rotating rigid body system or rotating particle system, the radial movement of some of its internal matter will inevitably lead to non-conservation of angular momentum.

According to the new law of motion [1], it can also be proved that the angular momentum of the complex rotating matter system is not conserved. According to the definition of angular momentum [6], angular momentum is:

$$
l=r \cdot p=r \cdot m \cdot u
$$

That is, the angular momentum is the product of the position vector of an object and its linear momentum.

And according to the new third law of motion [1], in most cases the momentum is not conserved in the interaction of objects. When a part of the substance on a rotating body moves radially, the linear velocity of the moving substance and the linear velocity of the unmoving substance will conflict. Therefore, a force of action will be formed between the two, and according to the new third law of motion [1], it is inevitable that momentum will not be conserved, that is, linear momentum will not be conserved. The change of linear momentum leads to the non-conservation of angular momentum.

Therefore, in addition to the circular motion of a single substance or particle, this very simple rotational motion can satisfy the law of conservation of angular momentum. In most complex rotating matter systems or rotating particle systems, angular momentum is generally not conserved. The law of conservation of angular momentum does not apply. Therefore, the law of conservation of angular momentum is applicable in some specific cases, but not in other more cases.

\section{Summing-up}

In most material interactions, momentum is not conserved. In most complex rotating matter or particle systems, angular momentum is also not conserved. According to the principles of non-conservation of momentum and non-conservation of angular momentum, science is bound to undergo tremendous changes.

\section{Acknowledgments}

Thanks to the Editorial Office. Thanks to the authors of the references.

Thanks to my teachers for my strong support for my scientific and technological activities: Professor Shixu Guan, Chief editor Xinmin Zhu, President Lanxu Xu.

Thanks to the universities that have helped me: Department head Shuquan Wang, Department head Xinde Jiang, Asso- 
ciate Professor Risheng Piao and Many teachers.

Thanks to the scientific and technical worker, experts and scholars, given me many of help.

\section{References}

[1] New Newtonian mechanics and new laws of motion, GuagSan Yu. http://vixra.org/abs/1507.0025 [2016-12-07 21:54:20].

[2] The experiment of the Inertia-torque, GuagSan Yu. http://blog.sina.com.cn/u/2100834921 [2014-02-23 13:25].

[3] Analyze Mistake of the Newton Third Law, GuagSan Yu. http://vixra.org/abs/1409.0115v2 [2014-09-14 23:22:57].

[4] The Inverse Derivative-The new algorithm of the derivative, GuagSan Yu. http://vixra.org/abs/1601.0189 [2016-09-26 21:24:48].

[5] New Rotational Dynamics - Inertia-Torque Principle and the Force Moment the Character of Statics, GuagSan Yu, http://vixra.org/abs/1411.0098 [2015-02-02 21:17:02].

[6] D. Halliday, R. Resnick. (1979). Physics foundation. Zeng Yongling. Beijing: Higher Education Publishing Organization. 